Annuaire suisse de politique de développement

18 | 1999

La Suisse et l'action humanitaire

\title{
L'humanitaire dans la politique extérieure de la suisse
}

Jean F. Freymond

\section{(2) OpenEdition}

Édition électronique

URL : http://journals.openedition.org/aspd/666

DOI : 10.4000/aspd.666

ISSN : 1663-9669

Éditeur

Institut de hautes études internationales et du développement

\section{Édition imprimée}

Date de publication : 1 janvier 1999

Pagination : 25-37

ISSN : 1660-5934

\section{Référence électronique}

Jean F. Freymond, «L'humanitaire dans la politique extérieure de la suisse », Annuaire suisse de politique de développement [En ligne], 18| 1999, mis en ligne le 14 juillet 2012, consulté le 08 septembre 2020. URL : http://journals.openedition.org/aspd/666 ; DOI : https://doi.org/10.4000/aspd.666 


\title{
L'HUMANITAIRE DANS LA POLITIQUE EXTÉRIEURE DE LA SUISSE
}

\author{
JEAN F. FREYMOND*
}

A U DÉBUT D’AOÛT 1998, sur les bords du Congo, loin de la Berne fédérale, à des milliers de kilomètres des enceintes où se décide la politique extérieure de la Suisse, la folie des hommes a frappé, laissant derrière elle un cortège de désolation et d'horreurs, fruits d'une haine qu'on a éveillée: quartiers et infrastructures détruits, êtres humains innocents ou coupables poursuivis et qui se terrent pour ne pas trouver la mort atroce que ceux qui n'ont pas eu leur chance ont connue. Et depuis, des hommes et des femmes travaillent pour redonner un peu d'humanité à la vie de tous ces êtres qui se sont trouvés sur le passage de l'orage. Parmi ces hommes et ces femmes d'un peu partout, quelques-uns, représentants de la Suisse gouvernementale, diplomates, collaborateurs de la Direction du développement et de la coopération (DDC) et délégués du Comité international de la Croix-Rouge (CICR), qui, après avoir contribué à permettre à beaucoup de ceux qui se cachaient de s'en aller, ont entrepris de redonner espoir à un quartier entier en aidant, entre autres, à la reconstruction d'un hôpital. Cet exemple, parmi d'autres, est là pour rappeler d'emblée que la place de l'humanitaire dans la politique extérieure de la Suisse a plusieurs visages, que cet humanitaire se manifeste sur le terrain et que c'est là qu'il doit avant tout et en dernier ressort se concrétiser.

Cette place est importante aujourd'hui. L'humanitaire a commencé à l'occuper pour ainsi dire depuis la création de l'Etat fédéral en 1848, plus précisément depuis la création du Comité international de secours aux blessés ${ }^{1}$ en 1863 et l'invitation lancée par le Conseil fédéral aux Etats européens, aux Etats-Unis, au Brésil et au Mexique à prendre part au congrès qui se conclura, le 22 août 1864, par l'adoption de la première Convention de Genève pour l'amélioration du sort des blessés et des malades dans les armées en campagne.

La politique extérieure de la Suisse d'alors comprend deux composantes majeures: «une politique extérieure au sens étroit, limitée, empreinte d'une grande retenue, sans audace $\aleph^{2}$, dont la fonction essentielle est de préserver l'indépendance et la neutralité du pays. Peut-il en être autrement d'un petit Etat composite, à l'identité fragile, neutre de surcroît, dont la création récente est consécutive à des révolutions et une guerre civile? Ce pays ne manque cependant pas de courage quand il le faut. Affaire de Neuchâtel en 1856, dont l'objet est d'obtenir du roi de Prusse l'entière indépendance du canton de Neuchâtel, ou encore politique d'asile et des réfugiés en témoignent. Deuxième composante, «une politique technique dynamique dont le but est avant tout de permettre

* Directeur, Centre d'études pratiques de la négociation internationale, Genève. L'auteur tient à remercier ici Gudrun Reiner, qui s'est chargée d'une partie de la recherche que cette étude a impliquée.

1. Nom que se donne en 1863 ce qui deviendra le Comité international de la Croix-Rouge.

2. Jean F. Freymond, «Réflexions sur le champ des relations extérieures de l'Etat et la complexité du système international», Annuaire suisse de science politique 28/1988, p. 229. 
l'intégration du pays en tant qu'entité viable au sein d'un système européen et planétaire toujours plus interdépendant et complexe» ${ }^{3}$. Cette politique est souvent multilatérale. Elle se concrétise par une participation active de la Confédération aux grandes conférences internationales de caractère technique et par son adhésion dès leur création aux unions administratives, embryons des organisations internationales du $\mathrm{XX}^{\mathrm{e}}$ siècle ${ }^{4}$.

A ces deux composantes il faut en ajouter une troisième, distincte et d'une autre nature : une politique «humanitaire» ou de solidarité, fruit aux yeux de certains d'une «mission» impartie à la Suisse, par laquelle elle se singulariserait et qui lui conférerait une des dimensions de son identités. Au XIX ${ }^{\mathrm{e}}$ et presque jusqu'au milieu du $\mathrm{XX}^{\mathrm{e}}$ siècle, cette politique dont on trouve des échos dans la bouche de maints conseillers fédéraux ${ }^{6}$ relève d'abord du discours. Elle ne se traduit guère par un engagement systématique de l'Etat sous forme d'actions humanitaires ou plus généralement par un intérêt concret pour la chose humanitaire. Ainsi la création de la Croix-Rouge est avant tout l'œuvre du Comité de Genève dont les cinq membres, Henry Dunant en tête, se démènent, voyagent, agitent et convainquent. C'est particulièrement sur Paris que s'exercent les pressions. Napoléon III finit par appuyer l'idée d'une conférence diplomatique qui se tiendrait à Genève, mais dont il précise qu'il convient qu'elle soit convoquée par le Conseil fédéral, pour s'en tenir aux usages diplomatiques. Ce n'est qu'alors que le président du Comité de Genève, Gustave Moynier, se rend à Berne où il rencontre les conseillers fédéraux Jakob Dubs et Constant Fornerod et sollicite un appui qui est octroyé sans difficulté aucune ${ }^{7}$. De là date le rôle que la Confédération n'a cessé de jouer dans le développement du droit international humanitaire, un rôle dont le moteur a été et reste le Comité de Genève auquel Berne va prêter un appui répété et croissant. Cet appui se manifeste ainsi par la convocation en 1906 et 1929, à la suggestion du Comité, de nouvelles conférences diplomatiques dont sortiront les Conventions de Genève de 1906 et de $1929^{\circ}$.

Certes, de sa propre initiative, la Confédération s'engage sur le plan humanitaire, ainsi lors de l'internement de l'armée du général Bourbaki pendant la guerre franco-allemande de 1870-1871, lorsqu'elle accueille sur son sol des combattants grièvement blessés pendant la Grande Guerre et des dizaines de milliers d'enfants pendant la Seconde. Mais jusque tard dans le $\mathrm{XX}^{\mathrm{e}}$ siècle, les interventions humanitaires internationales de la Confédération sont avant tout sporadiques. Elles ne deviennent systématiques qu'à la fin de la Seconde Guerre mondiale, lors de la création en 1944, à l'initiative de la Confédération, du Don suisse en faveur des victimes de la guerre'. Le lancement du Don national procède en tout premier de la volonté de participer par un effort collectif, généreux et désintéressé, associant le peuple suisse à l'Etat, à la reconstruction de

3. Ibid.

4. Ibid. et Jean F. Freymond, «La politique extérieure multilatérale de la Suisse: aspects structurels», Relations internationales, no 32, hiver 1982, pp. 463-477.

5. Jean F. Freymond, «La politique extérieure multilatérale de la Suisse...».

6. Cf. Daniel Frei, Neutralität - Ideal oder Kalkül? Zweihundert Jahre aussenpolitisches Denken in der Schweiz, Verlag Huber, Frauenfeld, 1967.

7. Pierre Boissier, Histoire du Comité international de la Croix-Rouge. De Solférino à Tsoushima, Plon, Paris, 1963. On consultera aussi Documents diplomatiques suisses 1848-1945, vol. 1 (24.11.1848-30.11.1865), Benteli Verlag, Berne, 1990.

8. Cf. Pierre Boissier, Histoire du Comité international de la Croix-Rouge, et André Durand, Histoire du Comité international de la Croix-Rouge. De Sarajevo à Hiroshima, Institut Henry-Dunant, Genève, 1978.

9. Cf. Documents diplomatiques suisses 1848-1945, vol. 15 (8.9.1943-8.5.1945), Benteli Verlag, Berne, 1992. 
l'Europe. Mais il s'inscrit aussi dans le cadre d'une réflexion, menée pendant la guerre encore, sur la politique suisse d'après-guerre et sur les principes qui doivent fonder cette politique. La réinsertion dans la communauté des nations victorieuses d'une Suisse isolée, dont la neutralité est décriée et mal comprise, ne se présente pas sous les meilleurs auspices. La solidarité internationale, aux yeux de nombreux critiques, aurait dû se traduire par une participation de la Suisse à la guerre. A ce reproche, le Conseil fédéral - et ce sera là le mérite du chef du Département politique fédéral (DPF) d'alors, Max Petitpierre - répond en donnant un autre sens à cette solidarité qu'il couple étroitement avec la neutralité - l'une n'allant à bien des égards pas sans l'autre. "Le devoir essentiel lié à la neutralité, dira Max Petitpierre, est celui de solidarité. Il doit s'accomplir de deux manières selon les circonstances. Dans les périodes de guerre, les seules où la neutralité a sa raison d'être et déploie ses effets, la solidarité doit se manifester avant tout sur le plan humanitaire. Elle doit être en quelque sorte aveugle - de la même manière que la justice - et n'avoir d'autre but que de soulager, aider, atténuer. Elle est le corollaire d'un privilège: celui d'être épargné.

»En temps de paix ou dans ces époques incertaines comme celle où nous sommes aujourd'hui et qui ne sont pas de guerre mais sans qu'y règne la paix, la solidarité a un tout autre caractère. Elle implique la collaboration avec les autres pays en vue d'atteindre un même but par un effort commun. Cette solidarité n'est pas un noble sentiment: elle est la conséquence d'une interdépendance entre les peuples; elle est aussi la reconnaissance d'une responsabilité. Elle s'exerce au profit d'un intérêt collectif, dont participe tout Etat qui admet cette solidarité. $\gg^{10}$

L'après-guerre marque ainsi un tournant dans la politique humanitaire de la Suisse. D'occasionnelle qu'elle était, elle devient régulière. Dès 1946, les Chambres fédérales votent à plusieurs reprises des crédits affectés à l'aide humanitaire et, de surcroît, un appui substantiel au CICR. Cet appui se présente sous forme de contributions couvrant en moyenne la moitié du budget du siège de l'institution, dont l'origine remonte à 1931, et de contributions extraordinaires destinées à des actions spécifiques. La politique humanitaire ainsi se structure peu à peu. Les interventions se multiplient. Elles prennent parfois un caractère bilatéral et parfois se traduisent par un appui aux organisations internationales dont la vocation est humanitaire, dont les créations se succèdent depuis $1946^{11}$.

Autres tournants majeurs, la création du Corps suisse d'aide en cas de catastrophe (ASC), qui remonte à 1973, et surtout l'adoption en 1976 de la Loi fédérale sur la coopération au développement et l'aide humanitaire internationales. Désormais la politique humanitaire va prendre la forme qu'elle a de nos jours.

10. Max Petitpierre, «Allocution prononcée à Berne à un dîner de l'Association de la presse étrangère» le 23 novembre 1950, Max Petitpierre. Seize ans de neutralité active. Aspects de la politique étrangère de la Suisse (1945-1961), La Baconnière, Neuchâtel, 1980, p. 254.

11. «Message du Conseil fédéral à l'Assemblée fédérale à l'appui d'un projet de loi sur la coopération au développement et l'aide humanitaire internationales» du 19 mars 1973, pp. 16-19. 
$E^{\mathrm{N}}$ N CETTE FIN DU XX ${ }^{\mathrm{e}}$ siècle, l'humanitaire occupe toujours plus cette place de «pierre angulaire de la politique extérieure de la Suisse ${ }^{12}$. Il se veut explicitement l'expression d'une longue tradition spécifique associée souvent, mais pas exclusivement, à l'idée de la Croix-Rouge. Il est moins qu'autrefois le corollaire d'une neutralité "considérée comme impliquant des devoirs particuliers sur le plan humanitaire ${ }^{13}$ et qui doit conduire à chercher à alléger les souffrances dont on a soi-même été épargné. Il est enfin et surtout la manifestation de cette solidarité internationale qui constitue un des quatre principes de base sur lesquels repose explicitement, depuis la fin de la Seconde Guerre mondiale, la politique extérieure de la Confédération ${ }^{14}$. Tout d'abord une solidarité avec les victimes de catastrophes naturelles ou de ces désastres provoqués par l'homme, où que ces victimes se trouvent dans le monde, quelles que soient leur nationalité, leur race, leur religion ou leurs opinions, et dont l'aide humanitaire est le fer de lance. Mais une solidarité dont le champ est plus vaste, puisque la politique humanitaire au sens large vise à «protéger l'homme, son existence, sa dignité et sa liberté ${ }^{15}$.

Vaste programme qui soulève d'emblée la question de la définition de cette politique humanitaire au sens large. Le Conseil fédéral dans son «Message concernant la continuation de l'aide humanitaire internationale de la Confédération» du 25 mai 1988 précise ce qu'elle inclut: la promotion et la sauvegarde des droits de l'homme, le développement du droit international humanitaire, le soutien accordé au CICR, la politique internationale à l'égard des réfugiés, l'internement des prisonniers de guerre et l'aide humanitaire ${ }^{16}$. Il adopte ainsi une approche limitative et sectorielle, dont l'aide humanitaire, le soutien au CICR, le développement du droit international humanitaire et la politique des droits de l'homme sont les pièces maîtresses. Cette approche exclut entre autres de la politique humanitaire la coopération au développement et la politique d'asile - mais non la politique internationale à l'égard des réfugiés. En d'autres termes, la politique humanitaire stricto sensu ne constitue qu'un des volets de la politique de solidarité de la Confédération, au côté d'autres volets, la coopération au développement ou la politique d'asile par exemple. Cette politique humanitaire a pour dénominateur commun qu'elle vaut tout d'abord en temps de crises ou alors qu'une crise menace, qu'elle vise à prévenir et à alléger sur le champ la détresse des hommes et enfin qu'elle entend promouvoir la mise en pratique de valeurs et de règles humanitaires.

Le «Message du Conseil fédéral à l'appui d'un projet de loi sur la coopération au développement et l'aide humanitaire internationales » de mars 1973 est à cet égard explicite: «L'aide humanitaire et la coopération au développement constituent chacune pour leur part un aspect différent d'un même secteur particulièrement dynamique de notre politique de solidarité. ${ }^{17}$ Elles «se fondent toutes

12. «Message du Conseil fédéral concernant la continuation de l'aide humanitaire internationale» du 20 novembre 1996.

13. Pierre Micheli, «Les grandes lignes de la politique étrangère conduite par M. Max Petitpierre de 1945 à 1961 », Max Petitpierre. Seize ans de neutralité active, p. 33.

14. Joseph Boesch, «Epochen der Schweizerischen Aussenpolitik», Handbuch der schweizerischen Aussenpolitik, Paul Haupt, Bern, 1975, p. 120.

15. «Message du Conseil fédéral concernant la continuation de l'aide humanitaire internationale de la Confédération» du 25 mai 1988, p. 34.

16. Ibid., p. 35.

17. «Message du Conseil fédéral à l'Assemblée fédérale à l'appui d'un projet de loi sur la coopération au développement et l'aide humanitaire internationales» du 19 mars 1973, p. 5. 
deux sur le devoir des hommes à l'égard de leur prochain de ne pas s'accommoder de la détresse, de la misère et de l'indigence d'autrui, mais de contribuer dans la mesure du possible à les faire disparaître ${ }^{18}$. Et le Conseil fédéral poursuit en soulignant d'autres traits communs, ainsi la liaison étroite entre aide humanitaire et coopération au développement, dont il dit qu'elle peut «s'édifier sur des prestations de l'aide humanitaire et en prolonger les effets ${ }^{19}$.

Mais ces caractères communs ne peuvent faire oublier les nettes différences qui séparent coopération technique et aide humanitaire. Le fait tout d'abord que «l'aide humanitaire vise à pallier dans l'immédiat les effets d'une détresse profonde dans le cadre de conditions économiques et sociales données $»^{20}$ alors que « la coopération au développement a pour but de contribuer à modifier ces données en vue d'une amélioration durable des conditions de vie et d'attaquer ainsi le mal à la racine $»^{21}$. Cette détresse profonde et subite ne connaît pas de frontières. L'action humanitaire a donc un caractère universel et en principe peut toucher aussi bien les pays du Sud que les pays industrialisés. La coopération au développement est, elle, sélective. Elle s'adresse aux seuls pays en développement, et encore, à quelques-uns d'entre eux seulement.

Le législateur traite de la coopération au développement et de l'aide humanitaire internationales dans une même loi. Mais il prend bien soin de souligner ce qui les différencie, ainsi qu'il ressort dans les deux définitions qu'il adopte:

«La coopération au développement soutient les efforts des pays en développement en vue d'améliorer les conditions de vie de leurs populations. Elle doit contribuer à mettre les pays partenaires en mesure d'assurer leur développement par leurs propres forces. Elle tend, à long terme, vers un meilleur équilibre au sein de la communauté internationale. ${ }^{22}$

«L'aide humanitaire a pour objectifs de contribuer, par des mesures de prévention ou de secours, à la sauvegarde de la vie humaine lorsqu'elle est menacée ainsi qu'au soulagement des souffrances; elle vient notamment en aide aux populations victimes d'une catastrophe ou d'un conflit armé. $»^{23}$

Ce qui se reflète dans le fait que depuis lors, les messages du Conseil fédéral sur la continuation de l'aide humanitaire de la Confédération sont distincts de ceux par lesquels il sollicite le renouvellement des crédits-cadres destinés à assurer le financement de la coopération au développement.

Cette aide humanitaire, en quoi consiste-t-elle? Tout d'abord, elle représente un peu moins du cinquième du total des montants alloués à la coopération internationale de la Suisse, soit en moyenne près de 250 millions de francs. Mais ce chiffre doit être analysé de près car en grande partie, il consiste en contributions ordinaires et extraordinaires - parfois en assistance alimentaire - à un ensemble d'organisations internationales et d'organisations non gouvernementales. L'aide humanitaire de la Suisse passe ainsi en tout premier lieu par des institutions partenaires.

22. Loi fédérale sur la coopération au développement et l'aide humanitaire internationales du 19 mars 1976.

23. Ibid. 
Tableau n` 1: Aide humanitaire de la Confédération en 1996

\begin{tabular}{lrrrrr}
\hline & $\begin{array}{c}\text { Contributions } \\
\text { ordinaires }\end{array}$ & $\begin{array}{c}\text { Contributions } \\
\text { extraordinaires }\end{array}$ & $\begin{array}{c}\text { Assistance } \\
\text { alimentaire }\end{array}$ & $\begin{array}{c}\text { Intervention } \\
\text { directe ASC }\end{array}$ & Total \\
\hline $\begin{array}{l}\text { Intervention } \\
\text { directe ASC }\end{array}$ & & & & 26.5 & $\mathbf{2 6 . 5}$ \\
\hline $\begin{array}{l}\text { Organisations } \\
\text { internationales* }\end{array}$ & 23.2 & 34.8 & 22.3 & 2.2 & $\mathbf{8 2 . 5}$ \\
\hline $\begin{array}{l}\text { Organisations } \\
\text { Croix-Rouge* }\end{array}$ & 67.2 & 22.3 & 0.4 & & $\mathbf{8 9 . 9}$ \\
\hline $\begin{array}{l}\text { ONG suisses } \\
\text { Totaux }\end{array}$ & $\mathbf{9 0 . 4}$ & 18.2 & 13.3 & & $\mathbf{3 1 . 5}$ \\
\hline
\end{tabular}

Source: Rapport annuel de la Coopération au développement 1996.

* Le Programme alimentaire mondial (PAM) et le Haut-Commissariat des Nations Unies pour les réfugiés (HCR) reçoivent les trois quarts des montants versés aux organisations internationales.

** De ce montant, le CICR obtient 83,2 millions, le solde allant à la Croix-Rouge suisse.

Ce regard sur les chiffres est trompeur. Il pourrait donner l'impression que l'aide de la Confédération est essentiellement axée sur l'intervention indirecte de tiers, les interventions directes n'y jouant qu'un rôle secondaire. Ce serait minimiser l'importance de ces interventions directes dans lesquelles sont engagés les membres du Corps suisse d'aide en cas de catastrophe (ASC). Ces interventions sont intégrées dans une conception d'ensemble qui procède d'une stratégie claire articulée autour de quatre objectifs de prévention, de sauvetage, de survie et de reconstruction. Soit quatre stades qui appellent chacun des formes d'interventions distinctes: surveillance dans le but de prévenir; secours immédiats pour sauver; approvisionnement en eau, nourriture, abris et assistance médicale afin d'assurer la survie; et remise en état des infrastructures, première étape d'un processus de reconstruction dans lequel la coopération au développement est appelée à prendre le relais.

Deuxième grand volet de la politique de solidarité qui ne s'inscrit pas sous le chapeau de la politique humanitaire, la politique d'asile, traitée très longtemps distinctement de la politique internationale à l'égard des réfugiés qui, elle, est comprise sous ce chapeau. Aujourd'hui encore, même si une certaine collaboration existe, il subsiste un monde entre asile et politique internationale des réfugiés. Un monde qui tend peu à peu à se rapetisser ${ }^{24}$. Les raisons de ce fossé ne sont pas explicites. Elles se comprennent cependant. Ces deux grands domaines d'activités relèvent de deux départements différents. Jusqu'à récemment aussi, soit la toute fin des années 80 , l'afflux de réfugiés n'a guère soulevé les problèmes de toute nature qui font aujourd'hui la une des médias, ni n'a été la cause de dépenses qui ont passé de près de 150 millions de francs en 1986 à

24. Les remarques de Peter Arbenz sont à ce sujet très illustratives: «Pour le moins jusqu'à la crise yougoslave, la Direction de la coopération au développement et de l'aide humanitaire (DDA) a développé ses activités de politique internationale en matière de réfugiés pratiquement indépendamment de l'ODR et, lors de crises internationales, soutenait en premier lieu les organismes internationaux d'entraide compétents en l'espèce. En outre, avec son Corps pour l'aide en cas de catastrophe, elle réalisait ses propres projets et programmes. Les autres directions du DFAE responsables de la politique étrangère ont également mené pendant longtemps leurs propres politiques des droits de l'homme ou de coopération internationale indépendamment de la politique suisse en matière d'asile. Lorsque le Conseil fédéral a engagé en 1988 un Coordinateur en matière de politique internationale des réfugiés, la collaboration interdépartementale entre le DFAE et le DFJP s'est améliorée» (Peter Arbenz, Rapport sur une politique suisse en matière de migrations, mai 1995, p. 48) 
plus d'un milliard en 1997, et qui en 1998 pourraient dépasser le total des montants dépensés par la Confédération pour la coopération internationale ${ }^{25}$.

En dix ans donc, la situation a changé du tout au tout. Depuis 1996, les dépenses humanitaires de la Confédération - soit la somme de l'aide humanitaire et du coût de l'assistance aux réfugiés en Suisse - dépassent ses dépenses au titre de la coopération. Pour 1998, ces dernières devraient avoisiner 1050 millions de francs à mettre en regard des 1400 millions de l'assistance humanitaire prodiguée tant à l'extérieur qu'à l'intérieur du pays. C'est en effet la somme des efforts consentis qu'il s'agit de prendre en compte et non les seuls frais générés par l'aide apportée hors de Suisse. La Suisse se trouve ainsi - et elle n'est pas la seule - entraînée toujours plus dans un cercle vicieux humanitaire. Plus ses dépenses au titre de l'humanitaire progressent, plus les sommes qu'elle pourrait engager dans sa politique de coopération au développement plafonnent, pour ne pas dire diminuent. Or une bonne coopération constitue un des

Tableau n²: Coopération internationale de la Confédération et assistance aux réfugiés (1995-1997)

\begin{tabular}{lrrr}
\hline & $\mathbf{1 9 9 5}$ & $\mathbf{1 9 9 6}$ & $\mathbf{1 9 9 7}$ \\
\hline Coopération au développement & 972.9 & 976.8 & 943.8 \\
\hline Aide humanitaire & 244.8 & 230.4 & 222.1 \\
\hline $\begin{array}{l}\text { Apports de ressources aux pays } \\
\text { d'Europe centrale et orientale }\end{array}$ & 115.7 & 143.3 & 131.1 \\
\hline Total I (coopération internationale) & $\mathbf{1 3 3 3 . 4}$ & $\mathbf{1 3 5 0 . 5}$ & $\mathbf{1 2 9 7 . 0}$ \\
\hline Assistance aux réfugiés & 811.8 & 927.8 & 1016.5 \\
\hline Total II & $\mathbf{2 ~ 1 4 5 . 2}$ & $\mathbf{2 ~ 2 7 8 . 3}$ & $\mathbf{2 ~ 3 1 4 . 5}$ \\
\hline
\end{tabular}

Sources: Les budgets de I'ODR de 1986 à 1998, Office fédéral des réfugiés, Berne, 2.9.98; Rapports annuels de la Coopération au développement 1996 et 1997.

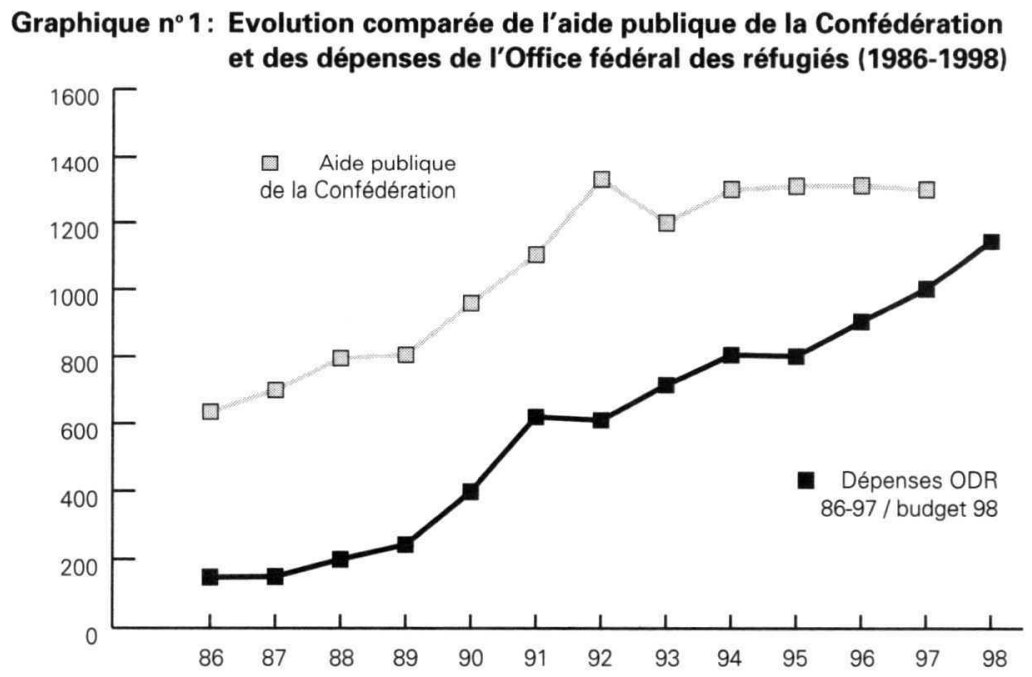

Sources: Les budgets de I'ODR de 1986 à 1998, Office fédéral des réfugiés, Berne, 2.9.98; Rapports annuels de la Coopération au développement 1996 et 1997; et autres.

25. Somme budgétée pour 1998 : 1'158'083'000 francs. Cf. Les budgets de l'ODR de 1986 à 1998, Office fédéral des réfugiés, Berne, 2.9.98. 
meilleurs instruments de prévention des crises humanitaires. Toute réduction de la coopération risque donc de contribuer à une multiplication de ces crises et à leur approfondissement.

En principe, la politique internationale à l'égard des réfugiés comporte d'abord toute une série d'activités qui touchent au droit international des réfugiés et à son application, et d'une manière générale à la pratique internationale en matière de réfugiés. Mais de toutes les dimensions de la politique internationale humanitaire de la Suisse, cette politique à l'égard des réfugiés présente une caractéristique majeure que les autres dimensions n'ont pas: elle est la seule qui est liée des plus étroitement à une problématique qui relève de la politique intérieure, la politique d'asile ${ }^{26}$. D'où le fait qu'en matière de politique de réfugiés, la Suisse se trouve devoir en premier lieu défendre ses intérêts avant que de promouvoir une approche dont l'objet serait de faire progresser les causes de l'humanitaire et des droits de l'homme.

Ce problème ne se pose guère en matière des droits de l'homme où, à quelques rares exceptions près, la Suisse ne se trouve pas sur la sellette, ni n'a à défendre sa pratique. La Suisse n'a explicitement de réelle politique en faveur des droits de l'homme que depuis le début des années 80, précisément depuis 1982, date à laquelle le Conseil fédéral définit sa politique dans son Rapport sur la politique suisse en faveur des droits de l'homme ${ }^{27}$. Le message à l'appui du projet de loi sur la coopération au développement n'évoque d'ailleurs pas le problème des droits de l'homme. Depuis, la promotion et la sauvegarde des droits de l'homme sont considérées formellement comme un des volets de la politique humanitaire ${ }^{28}$, mais un volet distinct, qui se situe sur un plan différent que les autres composantes de cette politique. Certes, «les conflits en tous genres, aux victimes desquels l'aide humanitaire est constamment amenée à prêter secours, s'accompagnent souvent de violations des droits de l'homme $»^{29}$, mais l'essence de l'humanitaire est finalement de s'attaquer en priorité à «sauvegarder des vies menacées et de soulager des souffrances $»^{30}$ par un engagement direct. L'action humanitaire est immédiate et concrète. L'action en faveur des droits de l'homme est plus générale. Elle déploie l'essentiel de ses effets dans le long terme et s'attache avant tout au développement de ces droits et à la promotion de mécanismes dont l'objet est d'en contrôler le respect. Parfois cependant, action humanitaire et sauvegarde des droits de l'homme se rejoignent. Ainsi lorsqu'il s'agit de protéger des personnes individuelles victimes de violation de ces droits $^{31}$.

La promotion et le développement du droit international humanitaire relèvent aussi formellement de la politique humanitaire de la Confédération, mais d'une manière peut-être plus explicite et plus directe que dans le cas des droits de l'homme, cela pour des raisons historiques tout d'abord. C'est la Confédération qui convoque la conférence qui adoptera en août 1864 la première Convention

26. Walter Kälin, «Die schweizerische Flüchtlings- und Asylpolitik», Nouveau Manuel de la politique extérieure suisse, pp. 761-775.

27. Rapport du Conseil fédéral sur la politique suisse en faveur des droits de l'homme du 2 juin 1982.

28. «Message concernant la continuation de l'aide humanitaire internationale» du 25 mai 1988, pp. 34-35.

29. Ibid., p. 43.

30. Ibid.

31. Mathias-Charles Kraft et Jean-Daniel Vigny, «La Politique suisse à l'égard des droits de l'homme», Nouveau Manuel de la politique extérieure suisse, Haupt, Berne, 1992, pp. 223-245. 
de Genève et c'est à cette date que remonte le rôle de la Suisse dans le développement du droit humanitaire. C'est la Confédération qui convoquera toutes les conférences diplomatiques successives. Deuxième raison, la responsabilité de la Confédération en tant qu'Etat dépositaire des Conventions de Genève et de leurs protocoles additionnels. Cette responsabilité lui confère des obligations. La Suisse est tenue de transmettre aux parties des informations sur les conventions et en particulier de porter à leur connaissance les ratifications et adhésions nouvelles, sans parler de certaines tâches qui lui sont confiées tant par les conventions que par les protocoles. Cette responsabilité paraît par ailleurs avoir incité la Confédération, plus sans doute que si elle ne l'avait pas assumée, à s'engager activement dans la promotion du droit humanitaire et son développement. Troisième raison enfin, qui touche à la matière. Le droit humanitaire est sans aucun doute bien plus proche de l'action humanitaire que ne le sont les droits de l'homme ${ }^{32}$.

Dernière des composantes de la politique humanitaire de la Confédération: son appui au CICR. Un appui qui prend plusieurs formes et qui, de quelque manière qu'on l'évalue, est très substantiel. Un appui d'ailleurs qui n'est pas à sens unique, car l'idée de la Croix-Rouge, les organisations qui ont été créées pour la promouvoir et les activités qui sont mises en œuvre en son nom ont contribué pour beaucoup à l'image d'une "Suisse humanitaire» et berceau de la CroixRouge, dont elle bénéficie et qui dans le même temps lui confère des obligations pour le moins morales. «Le Comité de Genève», lit-on dans un rapport du DPF de février 1944, «est une institution internationale envers laquelle notre pays a davantage de devoirs, mais pas plus de droits que n'importe quel Etat. ${ }^{33}$ Cinquante ans plus tard, cette affirmation garde toute sa valeur et reste le fondement de la politique de la Confédération qui a le souci de souligner constamment la nature sui generis de l'institution genevoise, son indépendance, son caractère non étatique, neutre et à vocation internationale. Cette neutralité, tant le CICR que Berne ne la veulent pas liée à la neutralité de la Suisse. Plus exactement, «la neutralité du CICR et celle de la Suisse sont complémentaires, elles ne se confondent pas ${ }^{34}$. Tous deux entendent qu'en aucune manière le CICR n'apparaisse comme un instrument de la politique extérieure de la Suisse, et en

32. Yves Sandoz, «La Suisse et le droit international humanitaire, en particulier les conventions de Genève pour la protection des victimes des conflits armés», Nouveau Manuel de la politique extérieure suisse, pp. 247-263.

33. «Proposition du Département politique au Conseil fédéral» du 14 février 1944, Documents diplomatiques suisses 1848-1945, vol. 15 (8.9.1943-8.5.1945), Benteli Verlag, Berne, 1992, p. 226.

34. «Message du Conseil fédéral concernant l'aide financière de la Confédération au budget siège du Comité international de la Croix-Rouge (1998-2001)» du 2 juin 1997, p. 74. Cette question de la neutralité et des neutralités respectives du CICR et de la Suisse a fait l'objet de nombreux débats et les opinions à ce sujet ont évolué. Rappelons pour l'histoire que la neutralité du personnel sanitaire constitue un des principes fondamentaux de l'idée Croix-Rouge, sur lequel s'accordent les participants à la conférence que convoque le Comité de Genève, à fin octobre 1863. Fait curieux, ce ne sont pas les membres du Comité qui tiennent le plus à cette neutralité, dont Gustave Moynier d'ailleurs ne souhaite pas qu'elle soit discutée, mais ceux des participants qui, ayant l'expérience de la guerre, ont pu constater que médecins et infirmiers, étant considérés comme des combattants, étaient de ce fait pris pour cibles, blessés ou tués. Démonstration une fois encore que les meilleurs juges de la valeur de la neutralité ne sont pas ceux qui l'ont faite leur, mais ceux qu'elle sert. Cf. Pierre Boissier, Histoire du Comité international de la Croix-Rouge, p. 107. Autre débat, datant d'une vingtaine d'années, qui s'inscrit dans le cadre des réflexions sur l'adhésion de la Suisse à l'Organisation des Nations Unies (ONU) : les répercussions d'une adhésion sur l'activité du CICR font l'objet d'une discussion approfondie au sein de la Commission consultative pour les relations de la Suisse avec l'ONU. Le CICR est invité à donner son avis, ce qu'il fait dans un mémorandum où il est dit que «l'action du CICR est essentiellement fondée sur la neutralité de la Suisse, que ce soit dans les guerres, les guerres civiles ou les troubles intérieurs... Grâce à cette neutralité, les belligérants ont une garantie supplémentaire de l'indépendance et de l'impartialité du CICR». Une opinion à laquelle se rallie la Commission. Cf. Rapport de la Commission consultative pour les relations de la Suisse avec l'ONU au Conseil fédéral du 20 août 1975, p. 55. 
particulier de sa politique humanitaire. Il y a communauté d'intérêts et concordance d'objectifs. Certaines responsabilités sont même partagées et obligent à une collaboration souvent étroite, particulièrement dans tout ce qui a trait à la diffusion et au développement du droit international humanitaire. Les liens tissés entre Berne et le Comité sont ainsi des plus étroits, avec la volonté farouche de part et d'autre de faire en sorte qu'à aucun moment le CICR ne perde cette indépendance indispensable à l'accomplissement de sa tâche. Cela quelle que soit l'importance de l'appui, particulièrement financier, que la Confédération octroie au CICR. Plus de 65 millions de francs sont inscrits au plan financier pour les années 1998 à 2000 et affectés au financement du budget du siège ${ }^{35}$, et un montant de près de 30 millions de francs devrait, bon an mal an, être versé à titre de contribution au budget extraordinaire de l'institution ${ }^{36}$. Soit une somme qui au total représente le tiers environ du montant dépensé par Berne dans le cadre de l'aide humanitaire et fait de la Confédération le troisième contributeur au budget du CICR, derrière les Etats-Unis et l'Union européenne.

Cette fresque rapide des grandes composantes de la politique humanitaire suggère quelques observations. Sans nul doute, l'approche choisie présente une certaine cohérence. Avoir regroupé un ensemble d'activités qui présentent des dénominateurs communs procède d'une logique qui s'explique, même si parfois on a l'impression que certaines de ces activités appartiennent à des champs bien distincts. Une des difficultés conceptuelles a pour origine le fait d'avoir voulu réunir sous un même chapeau la politique humanitaire au sens étroit, celle qui vise les situations de crise où il s'agit en toute urgence de sauver, et la politique humanitaire au sens large, dont l'objet est de "protéger l'homme, son existence, sa dignité et sa liberté $»^{37}$. Un but des plus noble qui se justifie entièrement, mais qui pose des problèmes opérationnels d'une tout autre nature que la politique humanitaire au sens étroit.

Autre problème, la confusion toute naturelle qui tend à se faire entre politique humanitaire et aide humanitaire. Là aussi, on est en présence d'un problème conceptuel né du fait qu'on a simplement juxtaposé de grandes composantes, sans trop s'interroger, non sur ce qu'elles avaient en commun, qui est évident, mais sur la manière de les articuler entre elles, d'en faire un tout qui prenne un sens sur le plan opérationnel.

Troisième observation, le fait que stricto sensu, coopération au développement et politique d'asile soient laissées en dehors de la politique humanitaire au sens large frappe. La logique voudrait qu'elles en soient au cœur, puisqu'il s'agit «de l'homme, de son existence, de sa dignité et de sa liberté». Comment expliquer ce fait? Une réponse a été esquissée plus haut en ce qui concerne la politique d'asile, à la fois problème de politique intérieure et relevant d'un autre département que le Département fédéral des affaires étrangères (DFAE) - sans parler du fait que le problème de l'asile n'explose que ces dix dernières années. Et pourtant, n'y a-t-il rien de plus humanitaire que la problématique des réfugiés et de l'asile? Quant à la coopération au développement, pour ne pas parler de la coopération avec l'Europe de l'Est qu'on tend à oublier, l'explication relève

36. «Message concernant la continuation de l'aide humanitaire internationale» du 20 novembre 1996.

37. «Message concernant la continuation de l'aide humanitaire» du 25 mai 1988, p. 34. 
sans aucun doute plus de l'histoire, de structures institutionnelles, de questions pratiques touchant aux demandes de financement qu'il est plus facile de sérier, que de la logique.

Ces problèmes conceptuels seraient sans grande importance s'ils n'avaient des répercussions pratiques. Les concepts ne sont pas innocents. Ils influent sur les structures, les modes de penser, de dire et de faire, la manière dont les institutions et les hommes collaborent ou s'ignorent. Ce qui à son tour se répercute sur la cohérence des politiques suivies, leur mise en œuvre et leur efficacité. Toutes choses d'ailleurs dont l'administration fédérale a conscience et auxquelles elle travaille.

[N CETTE FIN de siècle, l'humanitaire n'est plus ce qu'il était. La fin de la Eguerre froide avait donné à certains l'illusion de la fin de l'histoire. Or il s'avère que l'histoire n'a pas de fin. A l'espoir de temps meilleurs a succédé une prise de conscience que les défis auxquels l'humanité est confrontée ne font que gagner en ampleur et en complexité. Sur le seul plan de l'humanitaire au sens étroit, catastrophes naturelles et techniques se multiplient. Il en est de même des conflits de toute nature qui n'épargnent aucun continent.

Mais l'humanitaire n'est aussi plus ce qu'il était parce qu'il est devenu foire d'empoigne, objet d'enjeux, de controverses, de rivalités, de pouvoirs. Souvent il n'a pu éviter de se transformer en otage de la politique, en alibi et paravent de gouvernements qui, à l'abri de l'humanitaire, peuvent aussi bien masquer leur pusillanimité que se livrer à des pratiques qui contribuent à jeter de l'huile sur le feu. Les victimes elles aussi sont prises en otage. Certaines sont celles des causes qu'on épouse. Et il en est d'autres que n'épouse aucune cause, qui vivent leur tragédie, oubliées, loin des médias. L'humanitaire enfin draine des milliards et tout naturellement s'est souvent transformé en «business», quels que soient la foi et l'idéalisme qui animent ceux qui s'y engagent, particulièrement sur le terrain. Le phénomène ne date d'ailleurs pas de la fin de la guerre froide; il remonte à la guerre du Biafra et n'a fait que s' amplifier ${ }^{38}$.

C'est dans ce contexte nouveau que l'administration fédérale a commencé à se livrer à une réflexion dont le «Message du Conseil fédéral concernant la continuation de l'aide humanitaire internationale» du 20 novembre 1996 donne un bon reflet. Cette réflexion s'est poursuivie dans le cadre d'un Groupe de travail sur la politique humanitaire de la Suisse mis sur pied par la Division politique IV du DFAE (Politique des droits de l'homme et politique humanitaire) et dont le rapport final devrait être disponible sous peu. Parallèlement, tout au long des années 90 , on a assisté à des restructurations et à des changements de pratiques qui tous avaient pour objet de donner plus de cohérence et d'efficacité à la politique humanitaire. La création de la Division politique IV en est un exemple ${ }^{39}$.

38. L'humanitaire est à la mode. Il fait l'objet de livres nombreux dont certains illustrent bien ce qui est dit ici. $C f$. Mario Bettati, Le droit d'ingérence. Mutation de l'ordre international, Editions Odile Jacob, Paris, 1996. Ou encore Bernard Debré, L'illusion humanitaire, Plon, Paris, 1997.

39. Peter Arbenz, dans le rapport cité plus haut (en note 24), après avoir évoqué la création en 1988 du poste de coordinateur en matière de politique internationale des réfugiés, poursuit: «Les principales tâches du Coordinateur consistaient à sensibiliser le DFAE aux problèmes rencontrés par l'ODR lors de l'application de la politique d'asile et à 
Point de départ de la réflexion de l'administration, le Rapport sur la politique extérieure de la Suisse dans les années 90 du 29 novembre 1993 et les cinq objectifs de politique extérieure autour desquels le rapport s'articule:

- le maintien et la promotion de la sécurité et de la paix;

- l'engagement en faveur des droits de l'homme, de la démocratie et des principes de l'Etat de droit;

- l'accroissement de la prospérité commune;

- la promotion de la cohésion sociale;

- la préservation du milieu naturel.

Les responsables de l'aide humanitaire de la Confédération inscrivent délibérément cette aide dans le cadre défini par le rapport. Ils intègrent leur définition de l'action humanitaire dans un tout qui d'ailleurs ne comprend pas seulement la politique étrangère, mais aussi la politique de sécurité, la politique de développement et la politique de migration. Ils pensent complémentarité des politiques, des instruments et de l'action. Ils réfléchissent en termes de niches. Ils appréhendent avec justesse en quoi d'autres sont en mesure, dans la perspective de l'action humanitaire, de jouer un rôle central, particulièrement dans ce qui touche à la prévention des conflits et des catastrophes. Diplomatie préventive et bons offices, coopération en matière de vérification, de désarmement et de contrôle de trafics d'armes, lutte contre la pauvreté, engagement en faveur de la démocratie, des valeurs de l'Etat de droit, du respect des droits de l'homme et de la protection des minorités, lutte contre les causes des mouvements migratoires, tout est pris en compte dans la perspective d'une action humanitaire qu'il s'agit de soulager, tout à la fois en évitant par la prévention qu'on doive y avoir recours ou encore en l'appuyant si elle devient nécessaire. C'est là un tournant majeur dans la pensée. A l'approche sectorielle succède une vision qui procède d'un tout, la politique de l'Etat, tout d'abord sa politique extérieure certes, mais aussi sa politique intérieure, dans la mesure où les deux sont indissociables ${ }^{40}$.

Le rapport du Groupe de travail «Politique humanitaire» qui se prépare va plus loin encore. Il s'attache à conceptualiser la politique humanitaire d'une manière cohérente. Il en définit quatre objectifs: sauvegarde de la vie et de la dignité humaine en situation de crise aiguë, promotion des valeurs humanitaires, prévention et reconstruction. Puis ensuite, dans ce cadre, il précise la fonction des principales composantes de la politique humanitaire: développement et promotion du droit humanitaire et des droits de l'homme, activités d'aide et de coopération, activités de promotion de la paix.

Droit humanitaire et droits de l'homme se trouvent ainsi associés, tout en restant distincts, ce qui présente un sens dans la perspective des efforts en cours pour définir des «standards minimaux d'humanité». Il en est de même de l'aide humanitaire et de la coopération au développement, regroupées sous un même

amener le DFAE à mettre ses ressources diplomatiques à la disposition de l'ODR. Tel a surtout été le cas dans une phase où les forums internationaux pour les questions de la politique en matière d'asile et de migration ne cessaient de se multiplier. Cette collaboration a rendu possible la conclusion de différents accord bilatéraux de reprise de personnes et permis le dialogue avec les partenaires européens, dans l'intérêt de l'efficacité et de la crédibilité de la politique suisse de l'asile.

»Avec la réorganisation du DFAE approuvée par le Conseil fédéral à la fin de 1994, le Coordinateur est désormais rattaché à la Direction politique IV, nouvellement fondée; celle ci s'occupe, avec des effectifs renforcés, de la politique humanitaire, de la politique en matière des droits de l'homme et de la politique internationale des réfugiés" (Rapport sur une politique suisse en matière de migrations, p. 48).

40. «Message concernant la continuation de l'aide humanitaire internationale» du 20 novembre 1996. 
chapeau. Troisième volet enfin, les activités de promotion de la paix, sous la double forme de participations à des opérations de paix et de médiation ou, pour rester dans la continuité de la politique étrangère de la Suisse ${ }^{41}$, de bons offices, mais de bons offices renouvelés, adaptés à l'esprit, au contexte et à la pratique du temps.

Ce qui peut surprendre, c'est le fait que toute la problématique des réfugiés, des migrations et de l'asile ne soit pas intégrée dans ce concept. De bonnes raisons l'expliquent sans doute. Mais il paraît curieux que le volet de la politique humanitaire qui est source des plus grands problèmes et qui dévore des sommes équivalentes, bientôt peut-être supérieures, à tout ce qui est dépensé au titre de la coopération internationale de la Suisse soit évacué d'un concept qui veut avec raison prendre en compte toutes les composantes qui présentent un aspect humanitaire. En 1998, un milliard de francs, soit le double du montant alloué à la coopération technique bilatérale, aura été dépensé pour financer l'asile de 140 '000 personnes qui, à un titre ou à un autre, se trouvaient à fin août de cette année en Suisse. C'est là sans doute le plus formidable défi humanitaire que la Suisse doit aujourd'hui relever et qui ne pourra l'être qu'en commençant par l'intégrer dans un concept d'ensemble ${ }^{42}$.

L'humanitaire dans la politique extérieure de la Suisse est aujourd'hui au milieu du gué. Une vision, des objectifs, une stratégie sont en train d'être définis. C'est une étape essentielle. Le plus dur reste à faire, à savoir la mise en œuvre de cette stratégie, ce qui tout naturellement devrait obliger les uns et les autres à travailler autrement. A cet égard, il est souvent parlé de coordination. Mais cette coordination ne constitue pas une panacée; elle n'est que rarement acceptée par ceux qui sont coordonnés. Ce qu'il convient de promouvoir, c'est bien davantage la décentralisation des responsabilités, la circulation de l'information, le dialogue et la collaboration, et cela dans le cadre de projets communs qui appartiennent à tous. Là aussi la pratique est en mouvement, mouvement lent certes, mais révélateur des changements qui sont en cours. Le voyage conjoint des directeurs de la DDC et de l'ODR en ancienne Yougoslavie en est un bon exemple. La publication des Lignes directrices sur la Promotion des droits de l'homme et coopération au développement en est un autre ${ }^{43}$.

Sur les bords du Congo, les défis humanitaires s'accumulent. Bientôt peut-être, la nourriture viendra à manquer. Et la guerre là-bas, à l'autre bout du pays, se poursuit, hors de la vue des médias tenus à l'écart. Qu'elle est longue, la route qui va de Berne à Kinshasa, des concepts et des politiques à la réalité quotidienne de millions d'êtres humains qui n'aspirent qu'à une vie «normale» et que l'histoire ne cesse de broyer.

41. Cf. l'ouvrage classique sur les bons offices : Raymond R. Probst, «Good Offices» in International Relations in the Light of Swiss Practice and Experience, Martinus Nijhoff, Dordrecht, 1988.

42. La difficulté de la tâche est bien mise en lumière dans Marcus Cox, Strategic Approaches to International Intervention in Bosnia and Herzegovina, Centre for Applied Studies in International Negotiations (CASIN), Geneva, 1998.

43. Promotion des droits de l'homme et coopération au développement. Lignes directrices, DDC, 1997. 